\title{
Erector Spinae Block with Opioid Free Anesthesia in Cirrhotic Patients Undergoing Hepatic Resection: A Randomized Controlled Trial
}

\author{
Minatallah A Elshafie (D), Magdy K Khalil, Maha L ElSheikh, Nagwa I Mowafy \\ Department of Anesthesia and Intensive Care, National Liver Institute, Menoufia University, Sheeben Elkom City, Egypt
}

Correspondence: Minatallah A Elshafie, Department of Anesthesia and Intensive Care, National Liver Institute, Menoufia University, Sheeben Elkom City, Egypt, Tel +20 III 224 8935, Email menna80mas@gmail.com

Background: Hepatic resection is a major abdominal surgery with challenging pain management. We aimed to investigate the effect of erector spinae plane block (ESPB) with opioid free anesthesia (OFA) in cirrhotic patients scheduled for liver resection on perioperative pain management in terms of hemodynamic stability. Secondarily, we assessed time to first request for analgesia and perioperative fentanyl consumption, nausea and vomiting within 24 hours after surgery.

Methods: Forty patients were randomized to block group $(n=20)$ : OFA with ESPB and conventional group ( $n=20)$ : conventional balanced anesthesia with opioids (OFA associated non-opioid drugs [dexmedetomidine, magnesium sulfate, xylocaine, and acetaminophen] and ESPB). Bilateral ESP block was done with ultrasound guidance at the level of thoracic vertebrae $\mathrm{T}$ 6-7, the local anesthetic dose was $20 \mathrm{~mL}$ Bupivacaine $0.25 \%$ with adjuvant dexmedetomidine $(0.5 \mu \mathrm{g} / \mathrm{kg})$ on each side. We monitored hemodynamic stability as the primary endpoint (heart rate, mean arterial blood pressure, and cardiac output).

Results: Bilateral ESPB offered somatic and visceral analgesia for hepatic resection patients with no intraoperative fentanyl required. Postoperatively, the block group with dexmedetomidine adjuvant to the local anesthesia drugs showed delay in the first request for analgesia $(p=0.092)$ and decreased fentanyl requirement $(p<0.001)$, so no patient in the ESP group suffered from postoperative nausea and vomiting compared to $50 \%$ in the conventional group $(p<0.001)$.

Conclusion: Bilateral ESP block with OFA is an effective approach for intra- and postoperative analgesia in cirrhotic patients undergoing liver resection.

Keywords: hepatic surgery, cirrhosis, coagulopathy, pain management

\section{Introduction}

Hepatic resection is a major abdominal surgery with challenging pain management and a remarkable risk for postoperative pulmonary complications. It affects liver function, especially in cirrhotic patients with loss of functional hepatocytes. ${ }^{1}$ Epidural analgesia has shown decreased pain scores and side effects compared to systemic intravenous analgesics following open abdominal surgery. However, complications of epidural analgesia include a varying incidence of post-dural puncture headache, local anesthetic systemic toxicity, spinal hematoma, abscess, and hypotension which should be considered. Epidural catheter removal can also be delayed following liver resection due to postoperative coagulopathy. $^{2}$

Cirrhotic patients can benefit from opioid free anesthesia (OFA) as they are most sensitive to opioids' deleterious side effects. ${ }^{3}$ OFA is a multimodal procedure combining non-opioid drugs and regional anesthesia techniques that allow a better quality of anesthesia and patient satisfaction. ${ }^{4}$ The simultaneous use of non-opioid analgesic drugs (eg, dexmedetomidine, magnesium sulfate, xylocaine, and acetaminophen) can have a synergistic effect that improves postoperative analgesia and prevents opioid-related adverse effects (nausea, vomiting, ileus, sedation, and respiratory depression) while facilitating early movement. ${ }^{5}$ OFA can fulfill the implementation of enhanced recovery after surgery 
(ERAS) protocols designed for rapid recovery after major operations, supporting organ function, and decreasing stress response caused by surgical trauma. ${ }^{6}$

Erector Spinae Plane Block (ESPB) was first described in 2016 by Forero et $\mathrm{al}^{7}{ }^{7}$ as a regional anesthetic technique. Local anesthetic injected into this erector spinae fascial plane spreads in a craniocaudal fashion over several levels and to the thoracic paravertebral space, thereby targeting the dorsal and ventral rami of the spinal nerve. ${ }^{8}$ Thus, it can provide both somatic and visceral sensory blockade, making it an ideal regional anesthetic technique for abdominal surgery. The significant advantages of ESP block compared to epidural or paravertebral block relate to the relative ease, simplicity, safety of the block with fewer pneumothorax risks, and the risk of coagulation abnormalities usually suffered by the cirrhotic patient.

Dexmedetomidine is a potent $\alpha 2$ agonist and is a powerful adjuvant to regional anesthesia and analgesia. It can prolong the duration of the nerve block anesthesia resulting in increased effectiveness of the block in terms of duration, less use of opioids, and shorter hospital stay in the absence of clinically significant side effects (hypotension, nausea, vomiting, and pruritus)..$^{9,10}$

The primary goal of this study was to investigate the effect of ESP block with OFA in cirrhotic patients scheduled for liver resection on intraoperative and postoperative hemodynamics. The secondary objective was to assess the total intraoperative, postoperative fentanyl consumption, postoperative nausea and vomiting, and time to first request for analgesia according to $10 \mathrm{~cm}$ NRS for pain within $24 \mathrm{~h}$ after surgery.

\section{Materials and Methods}

All procedures performed in this study were in accordance with the institutional research committee's ethical standards of National Liver Institute, Menoufia University, and with the 1964 Helsinki Declaration and its later amendments or comparable ethical standards. All patients signed informed written consent approved by the local ethics committee at National Liver Institute, Menoufia University.

This was a randomized controlled trial which included 43 patients undergoing hepatic resection. Approval was obtained from the Institutional Review Board and ethics committee of the National Liver Institute, Menoufia University, Egypt (NLI IRB 00234/2020). This trial was prospectively registered with the Pan African Clinical Trial Registry (PACTR202101816486275) on January 26, 2021 and started on January 27, 2021, and ended on April 14, 2021. Registry URL; https://pactr.samrc.ac.za/TrialDisplay.aspx?TrialID=14621.

Patients were randomized into two groups, using a random number generator in sealed opaque envelopes. One group received opioid free anesthesia with erector spinae plane block $(n=22)$ where two patients were excluded from the analysis due to hemodynamic instability. The conventional group received conventional balanced anesthesia with opioids $(n=21)$ and patient was excluded from the analysis due to uncontrolled hypertension as the patient had undiagnosed hypertension.

Patients were aged 18-65 years, of both sexes. The inclusion criteria were cirrhotic candidates for hepatic resection with Child classification (A). Exclusion criteria were applied to patients with pre-existing significant acute liver failure. Patients who suffered from major intraoperative events (eg, severe hemodynamic instability, massive blood loss, massive blood products transfusion), patients who were allergic to any of the study drugs, those with opioid addiction, body mass index higher than $40 \mathrm{~kg} / \mathrm{m}^{2}$ and unwilling to participate in the study were excluded.

Anesthesia monitoring included a standard for basic anesthetic monitoring: fraction inspired oxygen concentration, fraction inspired and expired anesthetic agent, continuous invasive blood pressure monitoring (IBP; $\mathrm{mm} \mathrm{Hg}$ ), continuous central venous pressure monitoring $\left(\mathrm{cmH}_{2} \mathrm{O}\right)$, and urine output ( $\mathrm{mL} /$ hour). Electrical Cardiometry (EC) monitor (Osypka Medical GmbH, Berlin, Germany) was used for corrected flow time (FTc)-guided perioperative fluid optimization and cardiac output (L/min) monitoring. The Bispectral Index (BIS) monitor (Aspect Medical Systems Inc., Newton, MA, USA) was used for anesthesia depth monitoring.

\section{Anesthetic Technique}

After pre-oxygenation using $\mathrm{O}_{2} / \mathrm{Air}$ mixture $\left(\mathrm{FiO}_{2}=0.8\right)$ for 3-5min, in the conventional group, general anesthesia was induced with an intravenous dose of Propofol $(1.5-2 \mathrm{mg} / \mathrm{kg})$, Fentanyl $2 \mu \mathrm{g} / \mathrm{kg}$ and Rocuronium administered $(0.9 \mathrm{mg} / \mathrm{kg})$ 
to facilitate tracheal intubation. Then opioids were used in the maintenance of anesthesia to maintain appropriate hemodynamics.

In the block (ESPB) group, anesthesia was started with a dose of Propofol $(1.5-2 \mathrm{mg} / \mathrm{kg})$, Rocuronium $(0.9 \mathrm{mg} / \mathrm{kg})$, Xylocaine $60 \mathrm{mg}$, and Magnesium sulfate $1 \mathrm{gm}$ was infused with the induction and Dexmedetomidine infusion $(0.7 \mu \mathrm{g} / \mathrm{kg} / \mathrm{h})$ followed by tracheal intubation. For maintenance, Dexmedetomidine infusion $(0.2-0.7 \mu \mathrm{g} / \mathrm{kg} / \mathrm{h})$ was used to obtain appropriate hemodynamics. Then, bilateral erector spinae plane block guided by ultrasound at the level of thoracic vertebrae (T 6-7) was done. The local anesthetic dose was $20 \mathrm{~mL}$ Bupivacaine $0.25 \%$ with additive Dexmedetomidine $(0.5 \mu \mathrm{g} / \mathrm{kg})$ on each side.

In all patients, mechanical ventilation was initiated using pressure regulating volume-target mode, and ventilator settings included a TV of $6-8 \mathrm{~mL} / \mathrm{kg}$ of ideal body weight with the optimum PEEP for each patient. It was performed in all patients using a semi closed system (Datex Ohmeda GE, USA) adjusted to keep $\mathrm{SaO}_{2}>95 \%$ and end-tidal $\mathrm{CO}_{2}$ between $35-45 \mathrm{mmHg}$.

An intermittent bolus of Rocuronium $(0.15 \mathrm{mg} / \mathrm{kg})$ was administered to provide balanced general anesthesia according to the results of the train-of-four (TOF). Anesthesia was maintained with Sevoflurane in O2/Air mixture $(\mathrm{FiO} 2=0.4)$. End-tidal Sevoflurane concentration was adjusted to keep BIS between 40-60.

\section{Erector Spinae Plane Block Technique}

The patients were placed in the lateral position, then the skin was disinfected with $2 \%$ chlorhexidine in $70 \%$ alcohol, and a 2- to 5-MHz curved-array ultrasound transducer (SonoSite Edge, Bothell, Washington) was placed in a transverse orientation to identify the right lateral tip of the $\mathrm{T} 7$ transverse process corresponding to the inferior angle of the scapula. The transducer was then rotated 90 degrees into a longitudinal parasagittal orientation over the transverse processes to reach T5. The trapezius and erector spinae muscles were identified superficial to the acoustic shadow of the transverse processes. A 22-gauge, 80 -mm block needle (Pajunk, Geisingen, Germany) was inserted in a cephalad-to-caudad direction until the tip lay on the tip of the transverse process. Fluid, $0.5-1 \mathrm{~cm}$ saline, was injected to confirm correct needle tip position by visualizing spread under erector spinae muscle, then local anesthetic was injected.

\section{Analgesia in the ICU}

After surgery, all patients were transferred and stabilized in the ICU, then pain assessment was done according to the numerical pain score. When the patient showed a score of five or more, bolus fentanyl $0.5 \mu \mathrm{g} / \mathrm{kg}$ was given. After the first demand for analgesia and fentanyl bolus was given in the conventional group, a fentanyl infusion of $0.5 \mu \mathrm{g} / \mathrm{kg} / \mathrm{h}$ was maintained. In the block group, acetaminophen $1 \mathrm{~g} / 6 \mathrm{~h}$ was given, then with pain complaint (NRS score of five or more) a fentanyl bolus was given.

Measurement times during the perioperative course of liver resection were: baseline before anesthesia (T0), after induction of general anesthesia (T1), 10 minutes after the ESP block (T2), at the skin incision (T3), every one hour during the surgery (T4-6), postoperatively after transportation and stabilization in the ICU (T7) and after 2, 6, 12, 18 and 24 hours in the ICU (T8-12).

Demographic data included age (years), sex, and BMI $\left(\mathrm{kg} / \mathrm{m}^{2}\right)$. Systemic hemodynamics were heart rate (beat $\left./ \mathrm{min}\right)$, mean arterial blood pressure $(\mathrm{mmHg})$, and cardiometry derived variables of cardiac output $(\mathrm{CO})(1 / \mathrm{min})$. Intraoperative data included total intraoperative fentanyl consumption; catecholamine drug support requirements, blood product transfusion requirements, and time of surgery. Postoperatively patients were assessed at the first $24 \mathrm{~h}$ for; time to first request for analgesia according to the $10 \mathrm{~cm}$ numeric rating scale (NRS) for pain score, postoperative nausea and vomiting (PONV), and postoperative pulmonary complication (pneumothorax requiring chest tube placement).

\section{Statistical Methodology}

The required sample size was found to be 20 patients per group (number of groups=2) (total sample size $=40$ patients). ${ }^{11}$ The sample size was calculated using G Power version 3.1.9.2. ${ }^{12}$ The allocation sequence was generated using permuted block randomization technique and the block size was variable. Allocation sequence/code was concealed from the person allocating the participants to the intervention arms using sealed opaque envelopes. Double-blinded approach was adopted. Masking/blinding was employed to participants and statistical analysis team who were blinded to group allocation of patients. 
Data were collected and entered into the computer using SPSS (Statistical Package for Social Science) program for statistical analysis (ver 21). ${ }^{13}$ Data were entered as numerical or categorical samples by Friedman test. Pair-wise comparison, when Friedman test was significant, was carried out using Dunn-Sidak method. Z-test for independent proportions is used to compare two independent proportions. An alpha level was set to 5\% with a significance level of $95 \%$, and during the sample size calculation phase a beta error was accepted up to $20 \%$ with a power of study of $80 \%$, as appropriate. Comparisons were carried out between two studied independent not-normally distributed subgroups using Mann-Whitney $U$ test.

\section{Results}

Forty-three cirrhotic patients scheduled for liver resection were enrolled and randomized into the block group $(\mathrm{n}=22)$, where two patients were excluded from the analysis, and conventional group $(n=21)$ where one patient was excluded from the analysis. The Consolidated Standards of Reporting Trials (CONSORT) flow chart is presented in Figure 1.

The preoperative demographics were comparable, including age, sex and body mass index (BMI) (Table 1). The following data are presented as median values (IQR) for block versus conventional groups, respectively.

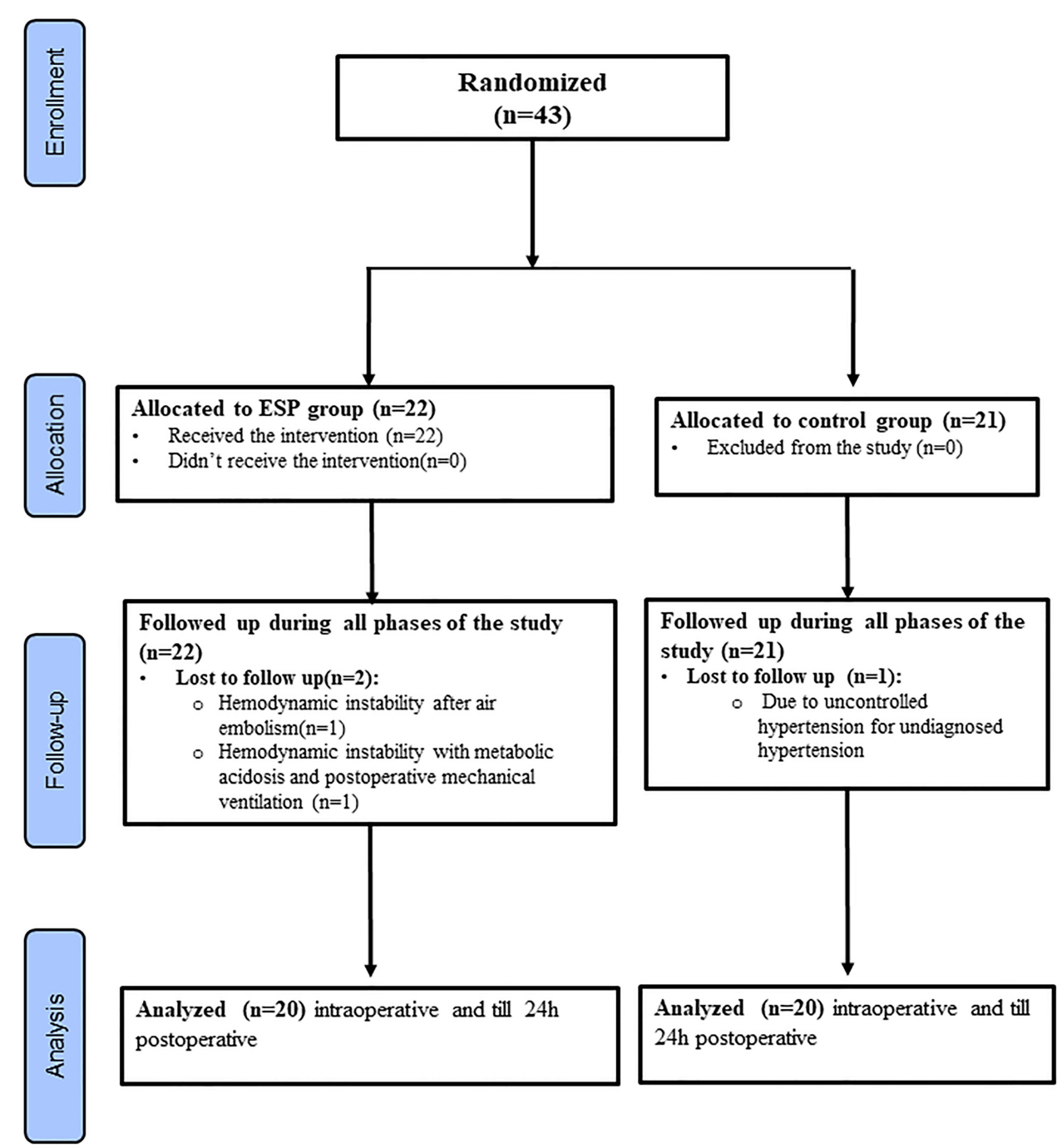

Figure I CONSORT flow diagram.

Notes: Adapted from: Schulz KF, Altman DG, Moher D, CONSORT Group. CONSORT 2010 Statement: Updated Guidelines for Reporting Parallel Group Randomised Trials. PLoS Med. 2010;7(3):el00025I. Copyright: (C) 2010 Schulz et al. Creative Commons Attribution License. ${ }^{28}$ 
Table I Demographic Data of Patients in the Block and Conventional Groups

\begin{tabular}{|c|c|c|c|}
\hline & Block Group $(n=20)$ & Conventional Group $(n=20)$ & Test of Significance \\
\hline \multicolumn{4}{|l|}{ Age (years) } \\
\hline -Min-Max & $21.00-63.00$ & $21.00-63.00$ & $Z_{(M W)}=0.138$ \\
\hline -Median (IQ-3Q) & $60.00(53.50-61.50)$ & $60.00(53.50-6 \mid .50)$ & $p=0.890 \mathrm{NS}$ \\
\hline \multicolumn{4}{|l|}{ Sex } \\
\hline -Male & $10(50.0 \%)$ & 7 (35.0\%) & $\chi_{(\mathrm{df}=1)}^{2}=0.921$ \\
\hline -Female & $10(50.0 \%)$ & $13(65.0 \%)$ & $p=0.337 \mathrm{NS}$ \\
\hline \multicolumn{4}{|c|}{ Body Mass Index (BMI) $\left(\mathrm{kg} / \mathrm{m}^{2}\right)$} \\
\hline -Min-Max & $19.00-36.00$ & $18.00-40.00$ & $Z_{(M W)}=0.665$ \\
\hline -Median (IQ-3Q) & $30.00(28.00-33.00)$ & $31.00(22.00-36.00)$ & $p=0.506 \mathrm{NS}$ \\
\hline
\end{tabular}

Abbreviations: $n$, number of patients; Min-Max, minimum - maximum; IQ-3Q, first quartile - third quartile; MW, Mann-Whitney test; $\chi^{2}$, Pearson Chi-squared test; df, degrees of freedom; NS, statistically not significant $(p \geq 0.05)$

Blood pressure preoperative level was higher in the block group than conventional group: 95.5 (87.0-100.5) versus 89.0 (81.0-93.0), and that was statistically significant $(p=0.019)$ although intraoperative BP was lower in the block group than the conventional group, and that was statistically significant except at $\mathrm{T} 1$ and $\mathrm{T} 2$ which were not statistically significant as shown in Figure 2.

Heart rate intraoperatively showed lower level in the block group than the conventional group, and that was statistically significant at T3 and T6: 75.5 (71.0-84.0) and 78.0 (73.5-85.0) versus 83.5 (80.5-87.5) and 85.5 (83.50$90.0),(p=0.021, p=0.015)$ respectively. Postoperative heart rate was also lower in the block group than the conventional group, and that was statistically significant at T8: 77.0 (74.0-87.0) versus 92.0 (77.5-95.5) ( $p=0.004)$ (Figure 3). Perioperative cardiac output measurements were not statistically significantly different between both groups $(p>0.05)$ (Figure 4).

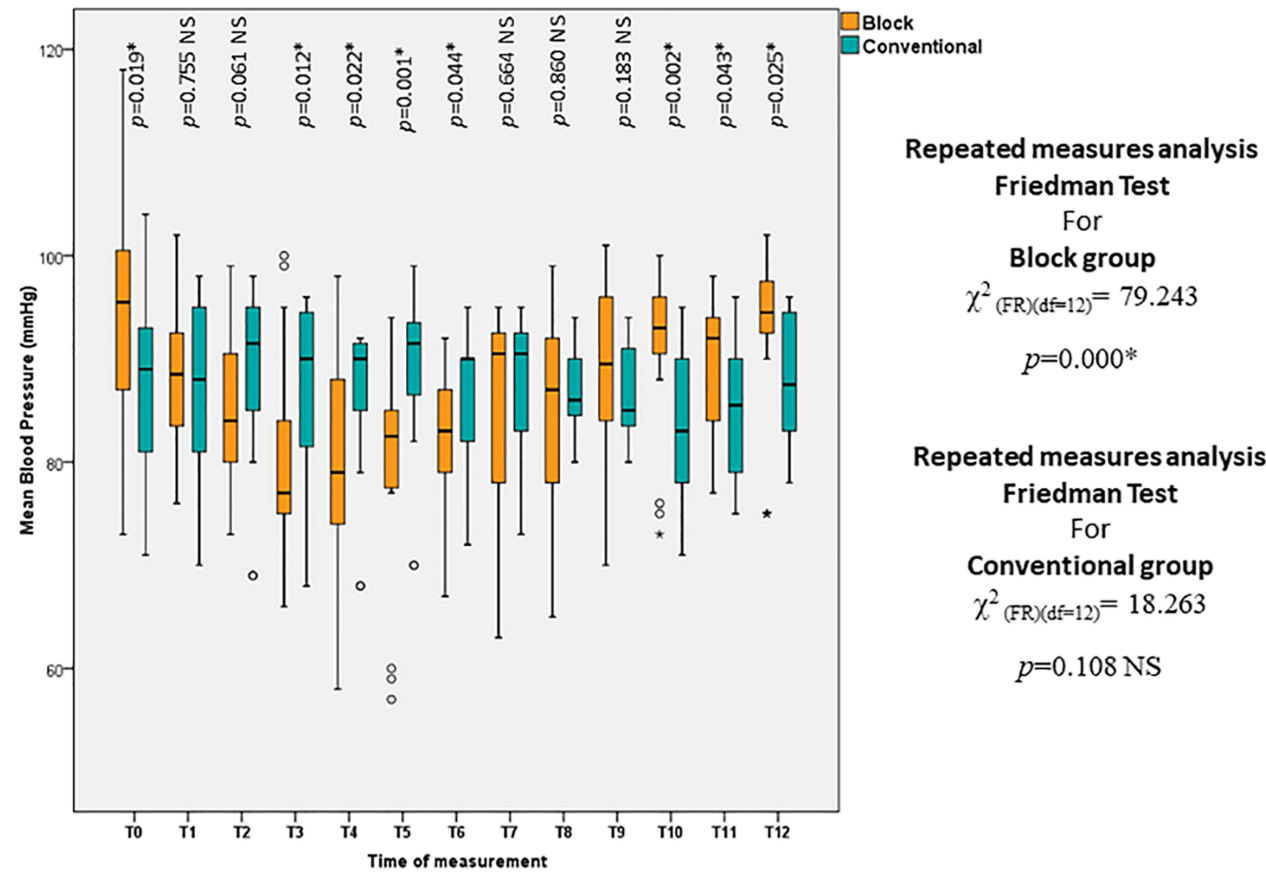

Figure 2 Box and whisker graph of mean blood pressure $(\mathrm{mmHg})$ in the studied groups, the thick line in the middle of the box represents the median, the box represents the interquartile range (from 25th to 75th percentiles), the whiskers represent the minimum and maximum after excluding outliers (circles) and extremes (black asterisks), the $*$ is for statistical significance $(p<0.05)$. 


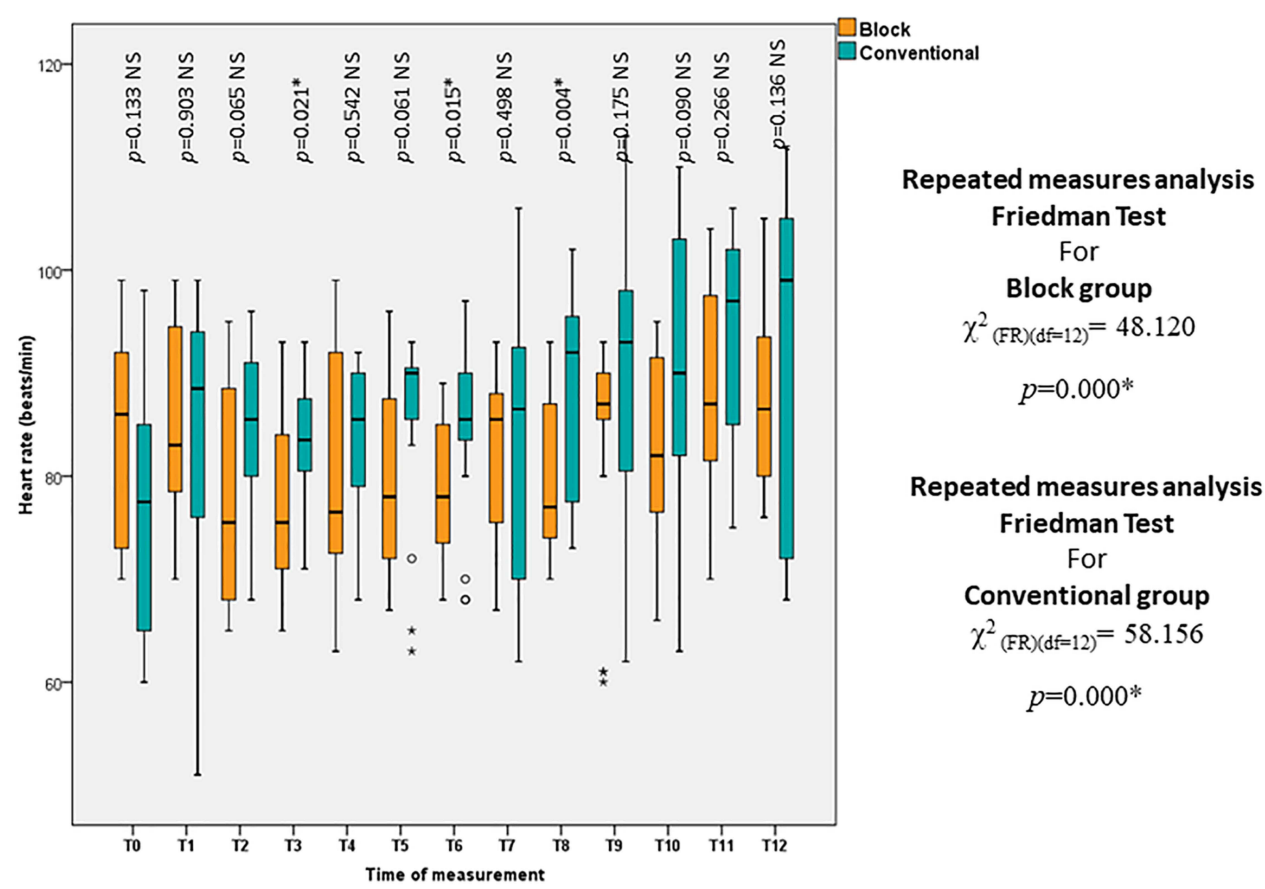

Figure 3 Box and whisker graph of heart rate (beats/min) in the studied groups, the thick line in the middle of the box represents the median, the box represents the interquartile range (from 25 th to 75 th percentiles), the whiskers represent the minimum and maximum after excluding outliers (circles) and extremes (black asterisks), the $*$ is for statistical significance $(p<0.05)$.

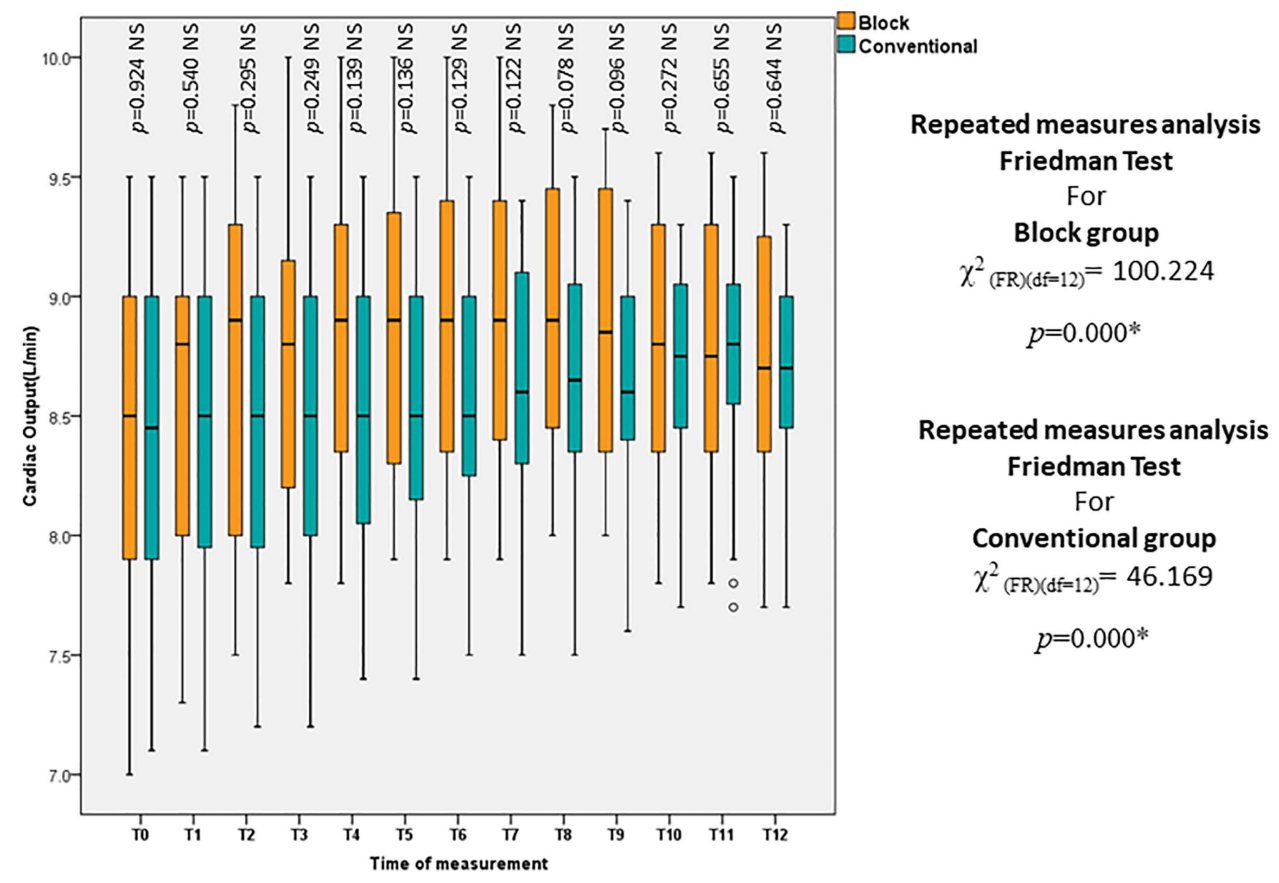

Figure 4 Box and whisker graph of cardiac output (L/min) in the studied groups, the thick line in the middle of the box represents the median, the box represents the interquartile range (from 25 th to 75 th percentiles), the whiskers represent the minimum and maximum after excluding outliers (circles) and extremes (black asterisks), the $*$ is for statistical significance $(p<0.05)$.

Intraoperative fentanyl consumption was not planned in the block group while in the conventional group its median was 500.0 (400.0-500.0) mic. Postoperative fentanyl consumption (mic) was statistically significantly lower in the block group compared with the conventional group: $50.0(50.0-80.0)$ versus $930.0(800.0-1150.0)$ respectively. The time (min) 
Table 2 First Time to Request Analgesia, Postoperative Fentanyl Consumption and Nausea and Vomiting in Block and Conventional Groups

\begin{tabular}{|c|c|c|c|}
\hline & Block Group $(n=20)$ & Conventional Group $(n=20)$ & Test of Significance \\
\hline \multicolumn{4}{|c|}{ First time to request analgesia (minutes) } \\
\hline -Min-Max & $540.00-1440.00$ & $15.00-30.00$ & $Z_{(M W)}=5.475$ \\
\hline -Median (IQ-3Q) & $1080.00(840.00-1170.00)$ & $27.50(15.00-30.00)$ & $p=<.00 I^{*}$ \\
\hline \multicolumn{4}{|c|}{ Postoperative fentanyl consumption (mic) } \\
\hline -Min-Max & $40.00-100.00$ & $400.00-1200.00$ & $Z_{(M W)}=5.458$ \\
\hline -Median (IQ-3Q) & $50.00(50.00-80.00)$ & $930.00(800.00-1150.00)$ & $p=<.00 I^{*}$ \\
\hline \multicolumn{4}{|c|}{ Postoperative nausea and vomiting } \\
\hline$-n$ & 0 & 10 & $Z=3.6515$ \\
\hline$-\%$ within group & $0.00 \%$ & $50.00 \%$ & $p=<.00 I^{*}$ \\
\hline$-\%$ within event & $0.00 \%$ & $100.00 \%$ & \\
\hline
\end{tabular}

Note: *Statistically significant $(p<0.05)$.

Abbreviations: $n$, number of patients; Min-Max, minimum - maximum; IQ-3Q, first quartile - third quartile; MW, Mann-Whitney test.

to the first request for analgesia postoperatively was statistically significantly longer in the block group versus the conventional group: 1080.0 (840.0-1170.50) versus $27.5(15.0-30.0)$ respectively $(p<0.001)$. The number of patients who suffered from postoperative nausea and vomiting was lower in the block group versus the conventional group $(0 / 20 \mathrm{vs}$ 10/20; $p<0.001$ ) (Table 2).

Both the need for intraoperative catecholamine and the median time of surgery (hours) were comparable between both groups ( $p=0.693, p=0.148$; respectively). No patient required blood products.

\section{Discussion}

Hepatic resection in cirrhotic patients is a major abdominal surgery with challenging pain management due to extended subcostal incision, rib retraction, as well as diaphragmatic and peritoneal irritation which have both visceral and somatic components ${ }^{14}$ with risk of coagulopathy. The role of epidural has been questioned due to the risk of epidural hematoma caused by the possible prolonged prothrombin time after liver surgery. ${ }^{15}$ It acts through sympathectomy resulting in hypotension and increased need for fluids and vasopressors.

Our patients who experienced ESP block with acetaminophen, magnesium sulphate, xylocaine, and dexmedetomidine as multimodal anesthesia with no opioids showed intraoperative hemodynamic stability in blood pressure, pulse and cardiac output when compared with the conventional method of anesthesia. Similarly, Fiorelli et $\mathrm{al}^{16}$ concluded that ESP blocks resulted in lower intraoperative opioid consumption, lower postoperative pain scores over $48 \mathrm{~h}$, decreased postoperative pulmonary complications, and increased patient satisfaction after open thoracotomy.

Maddineni et $\mathrm{al}^{17}$ reported a successful outcome of ESP as a replacement for gold standard epidural analgesia in achieving effective pain control following hepatic surgery. Similarly, a study by Gürkan ${ }^{18}$ has shown that US-guided ESP block and PVB provided adequate analgesia in patients undergoing breast surgery and have an opioid sparing effect by reducing morphine consumption.

The rare but dangerous risk of pneumothorax with the paravertebral block is much less of an issue with ESP block as this block showed great potential as part of an enhanced recovery protocol for major abdominal surgery. ${ }^{19}$ Other effective alternative blocks can provide both somatic and visceral pain relief, Rhomboid intercostal block, but the maximal spread with this approach is only between T2-T9, ${ }^{20}$ and Quadratus Lumborum block is technically more difficult, invasive, and may even result in motor weakness. ${ }^{21}$

This trial demonstrated effective results of ESP block on pain management among liver resection patients when combined with opioid free anesthesia. Forero et al in $2016^{7}$ described the ESP block as a new regional block to treat neuropathic thoracic pain. After that, the block has been used to manage acute and chronic pain with its ease of use and low complications for different surgeries. 
ESP block included local anesthetic injection below the erector spinae muscle on the transverse process which involves the spread in the paravertebral space leading to effective analgesia for somatic and visceral pain. It has shown a wide craniocaudal distribution of the local anesthetic. Nevertheless, many studies, such as Aponte et al's ${ }^{22}$ described that the injection of $20 \mathrm{~mL}$ of fluid at the $\mathrm{T} 7$ level determines diffusion from the upper thoracic vertebral segments to the lumbar ones.

We used the same volume of $20 \mathrm{~mL}$ of bupivacaine $0.25 \%$ on each side; it did not exceed referred toxic levels in our cirrhotic patients; Yassen et $\mathrm{al}^{23}$ used this LA dose, more safely, in transverse abdominis plane and rectus sheath space block in cirrhotic patients undergoing liver resection.

In our research, we added dexmedetomidine $(0.5 \mu \mathrm{g} / \mathrm{kg})$ on each side to the LA; we noted a prolonged time of the block and delayed first request for analgesia postoperatively with less pain intensity during the first $24 \mathrm{~h}$. Simultaneously Marhofer and Kettner ${ }^{24}$ demonstrated that dexmedetomidine effectively doubled the effectiveness and prolonged the peripheral nerve block to $60 \%$ with better postoperative analgesia and lower total PCA use during the first $72 \mathrm{~h}$ postoperatively.

Gao et al's ${ }^{9}$ study showed that the efficacy of dexmedetomidine is comparable to that of dexamethasone, which is already widely used as an adjuvant with regional anesthesia. It provides effective acute pain control resulting in less rescue analgesia and shorter hospital stays.

Our experience of ESP block with opioid free anesthesia was fruitful in reducing postoperative opiate analgesia requirement, reduced incidence of nausea and vomiting, and earlier discharge from ICU. In line with this, Piangatellia et $\mathrm{al}^{25}$ proved that a combination of ESP block on one side and TAP block on the other side was an effective approach for intra- and postoperative analgesia in parkinsonian patients undergoing laparoscopic right liver resection.

Our goal results matched Yao's ${ }^{26}$ findings that thoracic ESP block improved postoperative pain control and decreased breakthrough analgesic requirement, and also the results of Singh et $\mathrm{al}^{27}$ in reducing the incidence of nausea and vomiting, shorter stay in the ICU, and increased patient satisfaction.

One of our interesting findings were that in the Block group patients needed less end-tidal Sevoflurane concentration to keep BIS between 40-60. The other one we faced in the ICU was that patients after ESP at the level of T6-7 complained of slight epigastric pain that was relieved by acetaminophen and antispasmodic.

\section{Conclusion}

Bilateral ESP block with OFA is an effective approach for intra- and postoperative analgesia in cirrhotic patients undergoing liver resection regarding reduced opiate analgesia requirement, reduced incidence of nausea and vomiting, and no rebound pain in recovery with the delayed first request for analgesia. Future studies are required to investigate the ideal infusion regimen for continuous ESP blocks and which anesthetic and analgesic adjuvants should be included in that regimen.

\section{Data Sharing Statement}

Open access will be permitted. To get the data please send an e-mail to menna80mas@gmail.com. Researchers decided to send data when requested. No quality of request is required.

\section{Ethics}

IRB number: 00234/2020.

The Pan African Clinical Trial Registry number: (PACTR202101816486275).

\section{Funding}

The authors have no sources of funding to declare for this manuscript.

\section{Disclosure}

The authors declare no conflicts of interest for this work.

\section{References}

1. Bedawy A, Fayed N, Morad W, Madany S, Nematalah F, Yassen K. Rotational thrombo elastometry and standard coagulation tests for hepatic patients undergoing major liver resection. $J$ Anesth Clin Res. 2012;3(9):243. doi:10.4172/2155-6148.1000243 
2. Esteve N, Ferrer A, Sansaloni C, Mariscal M, Torres M, Mora C. Epidural anesthesia and analgesia in liver resection: safety and effectiveness. Rev Esp Anestesiol Reanim. 2017;64(2):86-94. doi:10.1016/j.redar.2016.06.006

3. Ziemann-Gimmel P, Goldfarb AA, Koppman J, Marema RT. Opioid-free total intravenous anaesthesia reduces postoperative nausea and vomiting in bariatric surgery beyond triple prophylaxis. Br J Anaesth. 2014;112(5):906-911. doi:10.1093/bja/aet551

4. Bakan M, Umutoglu T, Topuz U, et al. Opioid-free total intravenous anesthesia with propofol, dexmedetomidine and lidocaine infusions for laparoscopic cholecystectomy: a prospective, randomized, double-blinded study. Braz J Anesthesiol. 2015;65(3):191-199. doi:10.1016/j. bjan.2014.05.006

5. Chiu C, Aleshi P, Esserman LJ, et al. Improved analgesia and reduced post-operative nausea and vomiting after implementation of an enhanced recovery after surgery (ERAS) pathway for total mastectomy. BMC Anesthesiol. 2018;18(1):41. doi:10.1186/s12871-018-0505-9

6. Kamdar NV, Hoftman N, Rahman S, Cannesson M. Opioid-free analgesia in the era of enhanced recovery after surgery and the surgical home: implications for postoperative outcomes and population health. Anesth Analg. 2017;125(4):1089-1091. doi:10.1213/ANE.0000000000002122

7. Forero M, Adhikary SD, Lopez H, Tsui C, Chin KJ. The erector spinae plane block: a novel analgesic technique in thoracic neuropathic pain. Reg Anesth Pain Med. 2016;41(5):621-627. doi:10.1097/AAP.0000000000000451

8. Chin KJ, Forero M, Adhikary SD. Reply to Dr Ueshima and Dr Murouchi. Reg Anesth Pain Med. 2017;42(1):124-125. doi:10.1097/ AAP.0000000000000531

9. Gao Z, Xiao Y, Wang Q, Li Y. Comparison of dexmedetomidine and dexamethasone as adjuvant for ropivacaine in ultrasound-guided erector spinae plane block for video-assisted thoracoscopic lobectomy surgery: a randomized, double-blind, placebo-controlled trial. Ann Transl Med. 2019;7 (22):668. doi:10.21037/atm.2019.10.74

10. Andersen JH, Grevstad U, Siegel H, Dahl JB, Mathiesen O, Jæger P. Does dexmedetomidine have a perineural mechanism of action when used as an adjuvant to ropivacaine?: a paired, blinded, randomized trial in healthy volunteers. Anesthesiology. 2017;126(1):66-73. doi:10.1097/ ALN.0000000000001429

11. Charan J, Biswas T. How to calculate sample size for different study designs in medical research? Indian J Psychol Med. 2013;35(2):121-126. doi:10.4103/0253-7176.116232

12. Faul F, Erdfelder E, Lang A-G, Buchner A. G* Power 3: a flexible statistical power analysis program for the social, behavioral, and biomedical sciences. Behav Res Methods. 2007;39(2):175-191. doi:10.3758/BF03193146

13. IBM Corp. IBM SPSS Statistics for Windows, Version 21.0. Armonk, NY: IBM Corp; 2012.

14. Koul A, Pant D, Rudravaram S, Sood J. Thoracic epidural analgesia in donor hepatectomy: an analysis. Liver Transpl. 2018;24(2):214-221. doi:10.1002/1t.24989

15. Melloul E, Hübner M, Scott M, et al. Guidelines for perioperative care for liver surgery: Enhanced Recovery After Surgery (ERAS) Society recommendations. World J Surg. 2016;40(10):2425-2440. doi:10.1007/s00268-016-3700-1

16. Fiorelli S, Leopizzi G, Menna C, et al. Ultrasound-guided erector spinae plane block versus intercostal nerve block for post-minithoracotomy acute pain management: a randomized controlled trial. J Cardiothorac Vasc Anesth. 2020;34(9):2421-2429. doi:10.1053/j.jvca.2020.01.026

17. Maddineni U, Maarouf R, Johnson C, Fernandez L, Kazior MR. Safe and effective use of bilateral erector spinae block in patient suffering from post-operative coagulopathy following hepatectomy. Am J Case Rep. 2020;21:e921123. doi:10.12659/AJCR.921123

18. Gürkan Y, Aksu C, Kuş A, Yörükoğlu UH. Erector spinae plane block and thoracic paravertebral block for breast surgery compared to IV-morphine: a randomized controlled trial. J Clin Anesth. 2020;59:84-88. doi:10.1016/j.jclinane.2019.06.036

19. Hamilton DL, Manickam B. Erector spinae plane block for pain relief in rib fractures. Br J Anaesth. 2017;118(3):474-475. doi:10.1093/bja/aex013

20. Elsharkawy H, Saifullah T, Kolli S, Drake R. Rhomboid intercostal block. Anaesthesia. 2016;71(7):856-857. doi:10.1111/anae.13498

21. Wikner M. Unexpected motor weakness following quadratus lumborum block for gynaecological laparoscopy. Anaesthesia. 2017;72(2):230-232. doi:10.1111/anae. 13754

22. Aponte A, Sala-Blanch X, Prats-Galino A, Masdeu J, Moreno LA, Sermeus LA. Anatomical evaluation of the extent of spread in the erector spinae plane block: a cadaveric study. Can J Anaesth. 2019;66(8):886-893. doi:10.1007/s12630-019-01399-4

23. Yassen K, Lotfy M, Miligi A, Sallam A, Hegazi EAR, Afifi M. Patient-controlled analgesia with and without transverse abdominis plane and rectus sheath space block in cirrhotic patients undergoing liver resection. J Anaesthesiol Clin Pharmacol. 2019;35(1):58-64. doi:10.4103/joacp. JOACP_36_17

24. Marhofer D, Kettner SC, Marhofer P, Pils S, Weber M, Zeitlinger M. Dexmedetomidine as an adjuvant to ropivacaine prolongs peripheral nerve block: a volunteer study. Br J Anaesth. 2013;110(3):438-442. doi:10.1093/bja/aes400

25. Piangatelli C, Dalla Bona E, Tavoletti D, et al. Continuous erector spinae plane block for pain management in laparoscopic liver resection: case report. Revista Colombiana de Anestesiología. 2020;48(3):164-168.

26. Yao Y, Li H, He Q, Chen T, Wang Y, Zheng X. Efficacy of ultrasound-guided erector spinae plane block on postoperative quality of recovery and analgesia after modified radical mastectomy: randomized controlled trial. Reg Anesth Pain Med. 2019;45:5-9.

27. Singh S, Gunjan Kumar A. Ultrasound-guided erector spinae plane block for postoperative analgesia in modified radical mastectomy: a randomised control study. Indian J Anaesth. 2019;63(3):200. doi:10.4103/ija.IJA_758_18

28. Schulz KF, Altman DG, Moher D, CONSORT Group. CONSORT 2010 Statement: Updated Guidelines for Reporting Parallel Group Randomised Trials. PLoS Med. 2010;7(3):e1000251. 


\section{Publish your work in this journal}

Local and Regional Anesthesia is an international, peer-reviewed, open access journal publishing on the development, pharmacology, delivery and targeting and clinical use of local and regional anesthetics and analgesics. The journal welcomes submitted papers covering original research, basic science, clinical studies, reviews \& evaluations, guidelines, expert opinion and commentary, case reports and extended reports. The manuscript management system is completely online and includes a very quick and fair peer-review system, which is all easy to use. Visit http://www.dovepress.com/testimonials.php to read real quotes from published authors.

Submit your manuscript here: https://www.dovepress.com/local-and-regional-anesthesia-journal 